\title{
Genetic analysis in Italian families with inflammatory bowel disease supports linkage to the IBD1 locus - A GISC study
}

\author{
Vito Annese $^{1}$, Anna Latiano ${ }^{1}$, Paola Bovio ${ }^{1}$, Paola Forabosco ${ }^{2}$, Ada Piepoli ${ }^{1}$, \\ Giovanni Lombardi ${ }^{1}$, Arnaldo Andreoli ${ }^{3}$, Marco Astegiano ${ }^{4}$, Paolo Gionchetti ${ }^{5}$, \\ Gabriele Riegler ${ }^{6}$, Giacomo C Sturniolo ${ }^{7}$, Maurizio Clementi ${ }^{8}$, Eric Rappaport ${ }^{9}$, \\ Paolo Fortina ${ }^{9}$, Marcella Devoto ${ }^{2}$, Paolo Gasparini ${ }^{10}$ and Angelo Andriulli ${ }^{\text {P }}$ \\ ${ }^{1}$ Divisione di Gastroenterologia, Ospedale CSS-IRCCS, San Giovanni Rotondo, Foggia \\ ${ }^{2}$ Dipartimento di Oncologia Clinica e Sperimentale, Università di Genova, Facoltà di Medicina e Chirurgia, Genova \\ ${ }^{3}$ Divisione di Gastroenterologia, Ospedale N Regina Margherita, Roma \\ ${ }^{4}$ Dipartimento di Gastroenterologia, Ospedale Molinette, Torino \\ ${ }^{5}$ Istituto di Clinica Medica, Policlinico S Orsola, Bologna \\ ${ }^{6}$ Cattedra di Gastroenterologia, II ${ }^{a}$ Università di Napoli \\ ${ }^{7}$ Cattedra di Gastroenterologia, Università di Padova, Italy \\ ${ }^{8}$ Genetica Umana, Università di Padova, Italy \\ ${ }^{9}$ Department of Pediatrics, University of Pennsylvania School of Medicine and The Children's Hospital of \\ Philadelphia, USA \\ ${ }^{10}$ Servizio di Genetica Medica, Ospedele CSS-IRCCS, San Giovanni Rotondo, Foggia, Italy
}

\begin{abstract}
Epidemiological studies suggest that inherited factors influence susceptibility to inflammatory bowel disease (IBD), and some candidate loci have been described. In order to verify whether the same loci are responsible for predisposition to IBD in our population, we carried out a linkage study in a series of 58 Italian families with Crohn's disease (CD) and ulcerative colitis (UC). HLA-DQ alleles, motilin gene, and 34 microsatellites flanking the previously described loci on chromosomes 3, 6, 7, 12 and 16 were analysed by non-parametric linkage analysis in 16 and 23 families with $C D$ and $U C$, respectively, and in 19 families where $C D$ and $U C$ coexisted. Non parametric analysis using GENEHUNTER yielded maximum NPL scores for marker D16S408 in all IBD families combined $(2.71, P=0.003)$, for marker D16S419 in CD (1.97, $P=0.026)$ and for marker D16S514 in UC families $(2.44, P=0.007)$. These markers map in the previously described $I B D 1$ region. No significant linkage was found for markers of chromosomes 3, 6, 7 and 12. The present study performed in a Southern European population provides additional support for the conclusion that the IBDI locus has a clear role in the genetic susceptibility to IBD.
\end{abstract}

Keywords: inflammatory bowel disease; ulcerative colitis; Crohn's disease; linkage analysis; genetic predisposition

Correspondence: Vito Annese MD, Divisione di Gastroenterologia, IRCCS Casa Sollievo della Sofferenza, Via Cappuccini, 71013 San Giovanni Rotondo, Italy. Tel: +393388261812; Fax: + 39882411879 or 411705; E-mail: annesev@tin.it

Received 9 November 1998; revised 13 January 1999; accepted 19 January 1999 


\section{Introduction}

Crohn's disease (CD) and ulcerative colitis (UC) are common diseases in the Western world with an estimated prevalence rate of about 1:1000 inhabitants. ${ }^{1}$ Whilst their etiology remains elusive, epidemiological studies suggest that inherited factors might influence susceptibility to both diseases, especially to $\mathrm{CD}^{2}$ Although they are two distinct disorders, there is clinical and therapeutic overlap, which justifies their inclusion under the same heading of inflammatory bowel disease (IBD). Positive family history is the strongest risk factor so far identified for these diseases: given a proband with $\mathrm{CD}$ or UC, the relative risk of developing the same disease for first degree relatives is 10 and 8 , respectively. ${ }^{3}$ Moreover, the coexistence of both diseases in the same family is observed at a frequency substantially greater than that expected for each of them. This finding leads to the hypothesis that multiple genes contribute to both diseases and that phenotypic difference could result from the expression of specific genes. ${ }^{4}$

Hugot et al initially studied 25 families with CD and excluded linkage for the entire chromosome $6 .{ }^{5}$ In the attempt to disclose possible candidate genes, three genome scans have been undertaken. In two independent series of 41 and 71 European sib pairs with CD, Hugot et al identified a putative susceptibility locus (called IBD1) near marker D16S409 on chromosome 16 with a maximum lod score of $2.04{ }^{6}$ Satsangi et al performed a two-stage genome scan in two series of 75 and 85 families from the United Kingdom with a total number of 186 affected sib pairs. ${ }^{7}$ In this study, in which families with CD $(n=76)$, UC $(n=60)$, and mixed disease $(n=31)$, were included, strong evidence for the presence of susceptibility loci for both UC and CD was found on chromosome 3 (lod score 2.69), 7 (lod score 3.08), and 12 (lod score 5.47). In the same study linkage to D6S276, which lies close to the major histocompatibility complex on chromosome 6 , was excluded in CD families, whereas in UC the marker was more frequently shared by affected siblings. More recently a third genome-wide screen performed on 174 North American IBD families found evidence for linkage on chromosomes $1 \mathrm{p}, 3 \mathrm{q}$ and $4 \mathrm{q}$, and confirmed linkage to the pericentromeric region of chromosome $16^{8}{ }^{8}$

Confirmation of these initial studies in different populations is required in order to extend and test their meaning worldwide. However, replication studies have yielded contradictory results. Whilst most of them have confirmed the data for chromosomes 16 and 12 , none has so far been able to confirm linkage for chromosomes 3 and $7 .^{8-16}$

In this study we tested the presence of potential susceptibility loci on chromosomes 3, 6, 7, 12 and 16 in a series of Italian families with IBD.

\section{Materials and Methods}

In a multicentre programme of the GISC (Italian Group for the Study of the Colon and the Rectum) involving 12 centres throughout Italy, 85 families with one or more members affected by IBD were recruited. Patients were classified as having $\mathrm{CD}$, UC, or indeterminate colitis on the basis of clinical, endoscopic, radiological, and pathological data. ${ }^{17}$ In 22 families all affected members had CD, in $39 \mathrm{UC}$, and the remaining 24 had $\mathrm{CD}$ and UC (mixed families). In each family at least one unaffected relative was also available. All 85 families were included in the transmission disequilibrium analysis (see below). Families with one affected member or with parent/child affected pairs only were excluded as uninformative for the linkage analysis. Therefore, 58 families (16 CD, $23 \mathrm{UC}$ and 19 mixed) with two or more affected members other than parent/child were included in the linkage analysis. The type and number of affected relatives included in these families are shown in Table 1.

From each subject included in the study, peripheral blood was sampled after informed consent. The full purpose of the study was extensively explained to all participating subjects. Blood samples were collected into sodium citrate-containing tubes, frozen as a whole, and stored at $-30^{\circ} \mathrm{C}$ until extraction. Genomic DNA was then purified from peripheral blood leukocytes according to standard protocols. ${ }^{18}$ The 35 microsatellite markers, which were used to study five different regions on chromosomes $3,6,7,12$ and 16, are shown in Table 2. From chromosome 6, two further markers flanking the HLA region were added: DQ alleles (Amplitype HLADQ Perkin-Elmer, Norwalk, CT, USA) and the human motilin gene $(M L N)$. For each chromosome, the markers that gave the highest lod score in previous studies were included. Genetic maps and distances assumed in the multipoint analysis were taken from the Généthon database and from

Table 1 Characteristics of the IBD families included in the linkage analysis

\begin{tabular}{lrcc}
\hline Affected relatives & \multicolumn{3}{c}{ No of families } \\
& $C D$ & UC & Mixed \\
\hline 2 sibs & 10 & 18 & 11 \\
2 sibs +1 first cousin & 1 & - & - \\
2 sibs +1 aunt/uncle & - & 1 & 1 \\
3 sibs & 1 & - & 1 \\
4 sibs & 1 & - & - \\
1 aunt/uncle -1 niece/nephew & 2 & 1 & 4 \\
2 first cousins & - & 2 & 1 \\
1 grandparent -1 grandchild & 1 & 1 & 1 \\
Total & 16 & 23 & 19 \\
\hline
\end{tabular}


the Ceph/Généthon linkage map (http://www.cephb.f/ceph.genethon-map.html $)^{19}$ or from the Genetic Location Database (http://cedar.genetics.soton.ac.uk). The location of the human motilin gene $(M L N)$ on chromosome 6 was assigned close to the HLA-DQ $\alpha_{1}$ locus as previously described. ${ }^{20}$ Allele frequencies used in the genetic analysis were estimated by counting the observed alleles in unaffected, unrelated individuals.

For microsatellite markers, PCR was performed in a $15 \mu \mathrm{l}$ total reaction volume containing $1.5 \mu 110 \times$ Gene Amp PCR Buffer II, $1.5 \mu \mathrm{l}$ dNTPs $(2.5 \mathrm{~mm}), 1.5 \mu \mathrm{IgCl}_{2}(25 \mathrm{~mm}), 0.12 \mu \mathrm{l}$ Taq Gold polymerase $(5 \mathrm{U} / \mu \mathrm{l}), 0.5 \mu \mathrm{l}$ of each primer (5 to $15 \mathrm{pmol} / \mu \mathrm{l}), 50 \mathrm{ng} / \mu \mathrm{l}$ of genomic DNA template. Following $12 \mathrm{~min}$ at $95^{\circ} \mathrm{C}$ to activate the Taq Gold, amplification was achieved by the following protocol: 10 cycles at $94^{\circ} \mathrm{C}$ for $15 \mathrm{~s}$, $55^{\circ} \mathrm{C}$ or $56^{\circ} \mathrm{C}$ for $15 \mathrm{~s} ; 20$ cycles at $89^{\circ} \mathrm{C}$ for $15 \mathrm{~s}, 55^{\circ}-56^{\circ} \mathrm{C}$ for $15 \mathrm{~s}$ and $72^{\circ} \mathrm{C}$ for $15 \mathrm{~s}$. Final extension was at $60^{\circ} \mathrm{C}$ for $45 \mathrm{~s}$. PCR products were subjected to electrophoresis in $4.75 \% \mathrm{w} / \mathrm{v}$ polyacrylamide gels containing $8 \mathrm{M}$ urea, and run for 6 hours at $2500 \mathrm{~V}$ using automated $373 \mathrm{DNA}$ sequencer (PerkinElmer/Applied Biosystems Division, Foster City, CA, USA). Semi-automated DNA fragment sizing was performed using Genescan software (v.1.1), and genotyping by means of Genotype software (PE/ABD, Foster city, CA, USA, v.1.1).

Multipoint non-parametric linkage analysis was carried out with the GENEHUNTER program. ${ }^{21}$ This program was chosen because it performs non-parametric linkage analysis with a large number of markers in arbitrary pedigrees of moderate size. Non-parametric linkage analysis is carried out assessing marker allele sharing at each location of the genome among affected individuals and measuring whether they share

Table 2 List of polymorphic markers used and their distance

\begin{tabular}{ll}
\hline Chromosome & Marker list and map distance (cM) \\
\hline 3 & D3S3521 (4) D3S1076 (1) D3S3559 (5) \\
& D3S1573 (0.5) D3S1568 (5) D3S3717 (5) \\
& D3S1592 \\
& D6S260 (16) D6S464 (1) DQ 2 (0.5) MLN (1) \\
6 & D6S276 (13) D6S426 \\
7 & D7S484 (5) D7S2507 (7) D7S2428 (5) D7S519 \\
7 & (5) D7S506 (7) D7S2483 (7) D7S672 (7) \\
& D7S669 (7) D7S524 (7) D7S646 (5) D7S527 \\
12 & D12S368 (4) D12S1586 (6) D12S355 (1) \\
& D12S83 (3) D12S1702 \\
16 & D16S517 (1) D16S409 (1) D16S411 (9) \\
& D16S419 (8) D16S408 (7) D16S514 (1) \\
& D16S503 (3) D16S421
\end{tabular}

identical-by-descent alleles more often than expected under random segregation. Under the null hypothesis of no linkage and studying a large number of pedigrees, the non-parametric lod score (NPL) follows asymptotically a standardised normal distribution from which statistical significance is computed. Two nonparametric statistics are implemented: NPL-pairs (which assesses pairwise allele sharing among affected individuals) and NPL-all (which examines all affected individuals in the pedigree simultaneously and computes a score based on identity-by-descent sharing among all affected individuals). In our analysis we used the NPL-all statistics. Transmission disequilibrium test (TDT) was performed using the TDT multiallelic statistic included in the ANALYZE package. ${ }^{22}$ This test evaluates whether a marker allele is transmitted from parents to affected children more often than expected under random segregation. Therefore, TDT is a test of association between the disease and the marker alleles.

\section{Results}

Analysis of 58 families with IBD showed evidence of linkage for chromosome 16 but not for chromosomes 3 , 6, 7 and 12 .

\section{Chromosome 16}

Results of non-parametric multipoint analysis for eight chromosome 16 markers are shown in Table 3 and Figure 1. Identity-by-descent sharing among affected individuals was higher than expected under random segregation in the IBD sample in the whole region investigated. In the IBD families, a peak was obtained with marker D16S408 (NPL score $=2.70, \quad P$ value $=0.0035)$. Distribution of the NPL score in the $\mathrm{CD}$ and in the UC samples (Table 3 and Figure 1) was similar for markers D16S411, D16S419, and D16S408, but differed in the q-telomeric end of the region investigated, in which a NPL score of 2.44 was observed in the UC sample, whilst no significant distortion of allele sharing was observed in the CD sample. Specifically, the analysis of the CD-affected sample identified a $17 \mathrm{cM}$ region between markers D16S411 and D16S408, whereas in the UC families excess identity by

Table 3 Results of multipoint nonparametric linkage analysis using the NPL-all score of GENEHUNTER

\begin{tabular}{|c|c|c|c|c|c|c|c|c|}
\hline & \multicolumn{2}{|c|}{$\begin{array}{l}\text { IBD families } \\
(58)\end{array}$} & \multicolumn{2}{|c|}{$\begin{array}{l}C D \text { families } \\
\text { (16) }\end{array}$} & \multicolumn{2}{|c|}{$\begin{array}{l}\text { UC families } \\
\text { (23) }\end{array}$} & \multicolumn{2}{|c|}{$\begin{array}{l}\text { Mixed families } \\
\text { (19) }\end{array}$} \\
\hline & $\begin{array}{l}\text { NPL } \\
\text { score }\end{array}$ & $P$ value & $\begin{array}{l}\text { NPL } \\
\text { score }\end{array}$ & $P$ value & $\begin{array}{l}\text { NPL } \\
\text { score }\end{array}$ & $P$ value & $\begin{array}{l}\text { NPL } \\
\text { score }\end{array}$ & $P$ value \\
\hline D16S517 & 2.04980 & 0.020489 & 1.03509 & 0.150839 & 2.11397 & 0.016855 & 0.32112 & 0.376186 \\
\hline D16S409 & 1.97481 & 0.024479 & 1.23021 & 0.110581 & 1.85748 & 0.032273 & 0.29740 & 0.380952 \\
\hline D16S411 & 1.91618 & 0.028025 & 1.66423 & 0.049577 & 1.58546 & 0.056533 & 0.10784 & 0.457304 \\
\hline D16S419 & 2.20272 & 0.014037 & 1.97151 & 0.026088 & 1.81884 & 0.034360 & 0.07645 & 0.468463 \\
\hline D16S408 & 2.70793 & 0.003456 & 1.95452 & 0.026823 & 2.10631 & 0.017559 & 0.64785 & 0.259548 \\
\hline D16S514 & 1.87596 & 0.030701 & 0.41855 & 0.335655 & 2.44923 & 0.007045 & 0.20443 & 0.420375 \\
\hline D16S503 & 1.79552 & 0.036691 & 0.82937 & 0.203661 & 1.75080 & 0.040561 & 0.45849 & 0.325335 \\
\hline D16S421 & 1.50920 & 0.066090 & 0.00148 & 0.492133 & 2.05271 & 0.020339 & 0.37122 & 0.354732 \\
\hline
\end{tabular}


descent sharing was found over the whole region. No significant linkage was found in the mixed families.

TDT performed using the multiallelic statistic from the ANALYZE package was not significant for eight markers in 85 IBD families and in the subset of 39 UC families with one or more affected individuals. However, an overall $\chi^{2}$ of 4.64 ( $\left.1 \mathrm{df}, P=0.015\right)$ was found for marker D16S408 in 22 CD families, where allele 248 was transmitted 13 times from heterozygous parents to affected individuals as against three non-transmission.

\section{Other Chromosomes}

Results of non-parametric linkage analysis in our samples were not significant in the investigated regions on chromosomes 3, 6, 7 and 12 (all $P$ values gt; 0.05 ; data not shown). Multipoint NPL scores larger than 1 were observed in the UC sample at marker D6S426
$(\mathrm{NPL}=1.27)$ and at locus HLA-DQ (1.11) on chromosome 6; at markers D3S3521 (1.30), D3S1076 (1.20), D3S2559 (1.10), D3S1573 (1.16) and D3S3717 (1.06) on chromosome 3; at markers D7S519 (1.34), D7S2483 (1.43), D7S672 (1.28), D7S524 (1.01) D7S646 (1.42) and D7S527 (1.55) on chromosome 7. In the mixed sample NPL scores larger than 1 were observed at D12S368 and D12S1586 (1.39 and 1.12, respectively) on chromosome 12. All NPL scores were lower than 1 in the CD sample.

\section{Discussion}

The present study performed in a sample of Italian IBD families contributes additional evidence supporting the presence of a genetic locus on chromosome 16, which

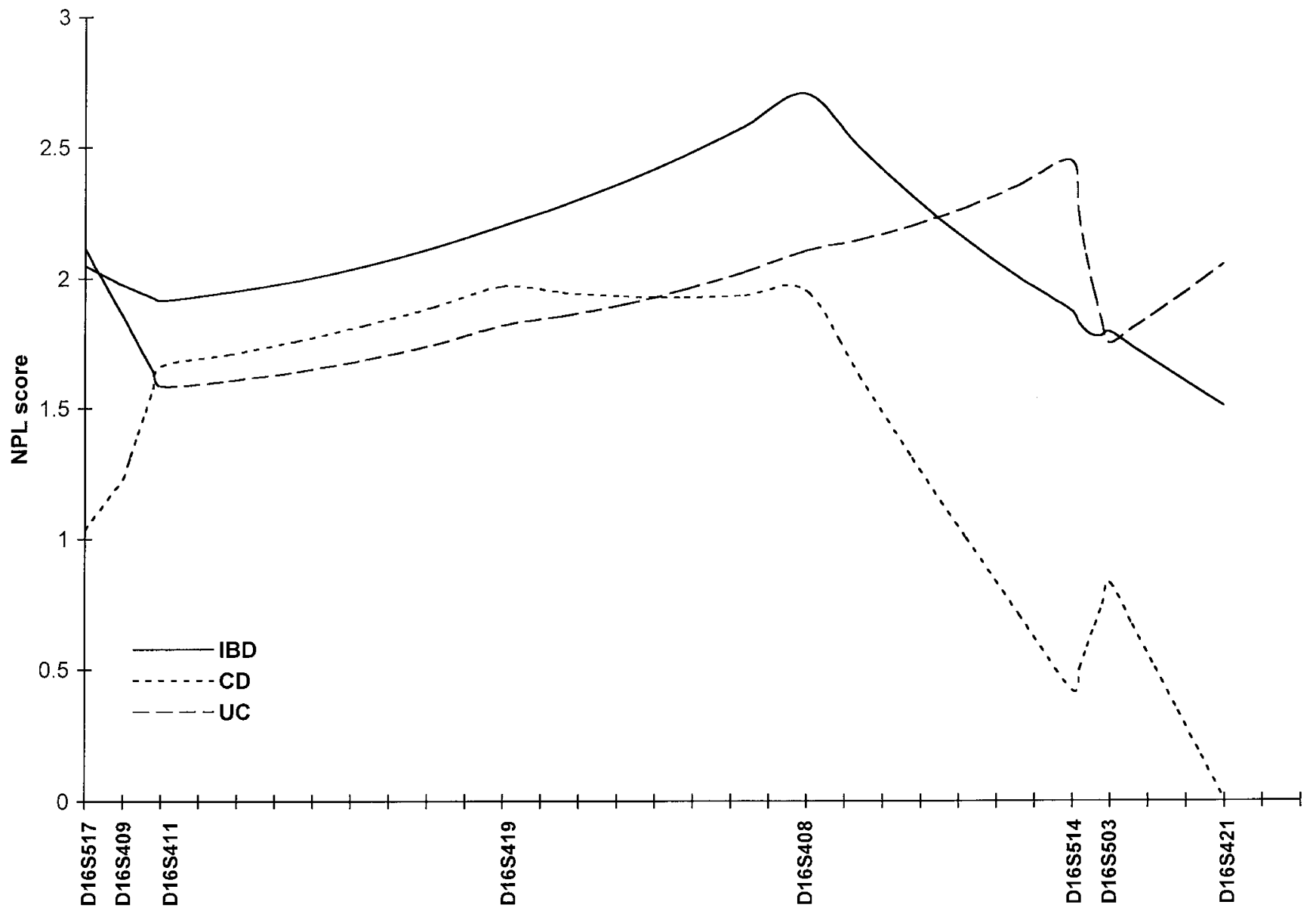

Marker loci $(1$ tick = $1 \mathrm{cM})$

Figure 1 NPL-all scores obtained with GENEHUNTER for the different subgroups of families with IBD, Crohn's disease and ulcerative colitis with markers of chromosome 16 
appears to encode for predisposing factors to IBD. Although a lod score of at least 3 is first required to establish linkage between a disease locus and a genetic marker, a lower threshold is sufficient to confirm a linkage that has already been found in an independent sample of families. ${ }^{23}$ Therefore, our results represent a statistically significant confirmation that a susceptibility locus for $\mathrm{CD}$, named $I B D 1$, is located in the pericentromeric region of chromosome $16 .{ }^{6}$ This region of the genome contains several candidate genes, including CD11 integrin, CD19, sialophorin and IL-4 receptor, which may be relevant to the pathogenesis of CD. Replication of the initial report has been undertaken in several studies carried out in different populations. Despite some negative reports ${ }^{15}$ most data confirmed that the IBD1 region contains a gene conferring predisposition to $\mathrm{CD}$, although disagreement exists about the precise location of this locus. ${ }^{8-11,14,16}$ Differences between studies may well be ascribed to the variability of the genetic background of the different populations so far evaluated, and to methodological limitations of linkage studies in fine mapping susceptibility loci for complex traits. ${ }^{24}$ In our CD families, NPL scores with associated $P$ value of less than 0.05 were obtained over the whole region encompassed by D16S411 and D16S408. A possible transmission disequilibrium was observed in our $\mathrm{CD}$ families at D16S408, where a multiallelic TDT produced a $P$ value of 0.015 .

Our study also reports positive linkage results for IBD1 in $23 \mathrm{UC}$ families. A recent study performed on a large sample of UC families also showed evidence supporting linkage to $I B D 1 .{ }^{13}$ Other three studies, which only included a small number of UC families, failed to show any significant result, ${ }^{9-11}$ whilst a fourth large sample of European UC families produced mainly inconclusive results. ${ }^{16}$ Overall, whether or not $I B D 1$ is also involved in predisposition to $\mathrm{UC}$ is still unclear. In our UC families, a peak NPL score of 2.44 $(P=0.007)$ was detected for D16S514. This locus is about 7 and $15 \mathrm{~cm}$ distant from D16S408 and D16S419, respectively, which gave the highest score in our CD families. However, there is considerable overlap in the regions of positive linkage in the $\mathrm{CD}$ and UC families, and difference in peak locations might be due to the relatively small number of families. Coexistence of linked and unlinked families in the UC sample may result in overestimation of the recombination fraction and therefore in mapping the disease locus away from its true location. In addition, it has been shown that a susceptibility gene for a complex trait does not necessarily lie in the region of maximum allele sharing, especially when the same gene confers only a modest increase in disease risk. ${ }^{24}$ Nonetheless, the possibility of two different loci on chromosome 16 contributing to susceptibility to CD and UC cannot be ruled out.

A putative gene associated with psoriasis has been recently reported on chromosome $16 .{ }^{25}$ Since psoriasis is more frequent in patients with Crohn's disease than in controls, ${ }^{26}$ it might well be the case that there is an immunomodulatory locus on chromosome 16 influencing the susceptibility to both diseases.

This study, in agreement with other reports, ${ }^{6,8,14,15}$ does not confirm previously reported linkage on chromosomes 3, 7 and 12. ${ }^{7}$ Multipoint NPL scores larger than 1 were observed over large intervals encompassing the previously reported loci on chromosomes 3 and $7^{7}$ in our UC families only, but all associated $P$ values were larger than 0.10 . This finding was not completely unexpected, based on the known difficulties in replicating linkage studies in complex traits. Linkage studies entail detection of weak effects that might be even less prominent in different studies, due to genetic heterogeneity of the disease combined with the ethnic variations of allele frequencies. Our non-significant results may just be the consequence of low power derived from our relatively small sample size.

There is considerable evidence supporting an association between some HLA loci and UC or in particular subgroups of UC patients. The HLA DRB1 and DQ $\beta_{1}$ genes have been recently found to influence UC susceptibility and disease behaviour. ${ }^{27,28} \mathrm{~A}$ significant increase of allele sharing by UC siblings for marker D6S276, which lies close to the HLA complex, was previously described. ${ }^{7}$ In the present study, we found a NPL score higher than 1.0 in the UC families at locus D6S426 $(\mathrm{NPL}=1.27)$ and marker DQ $(\mathrm{NPL}=1.11)$. Although these results were not statistically significant, the findings are in keeping with previous association studies $^{29}$ and further replication in larger series of patients may be warrant.

Finally, this study performed on an IBD population from the Mediterranean area contributes additional support to the overall conclusion that the IBDI locus on chromosome 16 has a clear role in genetic susceptibility to IBD. Further studies performed in larger samples will be necessary to establish definitely a role for other loci responsible for predisposition to IBD in our patients. 


\section{Acknowledgements}

The following are co-operative investigators in the Italian Group for the Study of Colon and Rectum (GISC). Gaetano Iaquinto, Italo Sorrentini (Servizio di Gastroenterologia, Avellino), Massimo Campieri, Stefano Peruzzo (Clinica Medica, Policlinico S Orsola, Bologna), Donatella Valpiani (Divisione di Medicina, Forli), Giuseppe Frieri, Maria Teresa Pimpo, Renzo Caprilli (Cattedra di Gastroenterologia, Università L'Aquila), Stelio Giaccari, Lucia Grasso (Divisione di Gastroenterologia, Galatina), Fabrizio Morace (Cattedra di Gastroenterologia, II ${ }^{a}$ Università di Napoli), Salvatore Cucchiara, Osvaldo Borrelli (Dipartimento di Pediatria, $I I^{a}$ Università di Napoli), Cosimo Prantera, Giustina Milite (Divisione di Gastroenterologia, Ospedale $N$ Regina Margherita, Roma), Renata D'Incà (Cattedra di Gastroenterologia, Università di Padova), Mario Rizzetto, Francesca Bresso (Dipartimento di Gastroenterologia, Ospedale Molinette, Torino), Angelo Pera, Maria Tilde Fiorentini (Divisione di Gastroenterologia, Ospedale Mauriziano, Torino) We thank the patients and their families who provided blood samples for this study and the AMICI Association (Associazione Italiana Malattie Inflammatorie Croniche dell'Intestino) for explaining the purposes of the study to members. This work was supported in part by a grant from the BYK Gulden, Italy and the Department of Pathology at the Children's Hospital of Philadelphia, USA.

\section{References}

1 Sandler RS: Epidemiology of inflammatory bowel disease. In: Targan SR, Shanahan F (eds): Inflammatory Bowel Disease. From Bench to Bedside. Williams \& Wilkins: Baltimore, 1994, pp 5-31.

2 Satsangi J, Jewell DP, Bell JI: The genetics of inflammatory bowel disease. Gut 1997; 40: 572-574.

3 Orholm M, Munkholm P, Langhoiz E, Nielsen $\mathrm{OH}$, Sorensen TIA, Binder V: Familial occurrence of inflammatory bowel disease. New Engl J Med 1991; 324: 84-88.

4 McConnell RB: Genetics of inflammatory bowel disease. In: Allan RN, Keighley MRB, Alexander-Williams J, Hawkins C (eds). Inflammatory Bowel Disease. ChurchillLivingston: London, 1990, pp 11-23.

5 Hugot JP, Laurent-Puig P, Gower-Rousseau C et al: Linkage analysis of chromosome 6 loci, including HLA, in familial aggregations of Crohns disease. Am J Med Genet 1994; 52: 207-213.

6 Hugot JP, Puig PL, Gower-Rousseau C et al: Mapping of susceptibility locus for Crohn's disease on chromosome 16. Nature 1996; 379: 821-823.

7 Satsangi J, Parkes M, Louis E et al: Two stage genomewide search in inflammatory bowel disease provides evidence for susceptibility loci on chromosomes 3, 7 and 12. Nat Genet 1996; 14: 199-202.

8 Cho JH, Nicolae DL, Gold LH et al: Identification of novel susceptibility loci for inflammatory bowel disease on chromosomes 1p, 3q, and 4q: evidence for epistasis between 1p and IBDI. Proc Natl Acad Sci USA 1998; 95: $7502-7507$.
9 Ohmen JD, Yang HY, Yamamoto KK et al: Susceptibility for inflammatory bowel disease on chromosome 16 has a role in Crohn's disease, but not in ulcerative colitis. Hum Mol Genet 1996; 10: 1679-1683.

10 Cho JH, Fu Y, Kirschner BS, Hanauer SB: Confirmation of a susceptibility locus for Crohn's disease on chromosome 16. Inflamm Bowel Dis 1997; 3: 186-190.

11 Parkes M, Satsangi J, Lathrop GM, Bell JI, Jewell DP: Susceptibility loci in inflammatory bowel disease. Lancet 1996; 348: 1588.

12 Duerr RH, Barmada MM, Zhang L et al: Linkage and association between inflammatory bowel disease and a locus on chromosome 12. Am J Hum Genet 1998; 63: 95-100.

13 Mirza MM, Lee J, Teare D et al: Evidence of linkage of the inflammatory bowel disease susceptibility locus on chromosome 16 (IBDI) to ulcerative colitis. J Med Genet 1998; 35: $218-221$.

14 Brant SR, Fu Y, Fields CT et al: American families with Crohn's disease have strong evidence for linkage to chromosome 16 but not chromosome 12. Gastroenterology 1998; 115: 1056-1061.

15 Rioux JD, Daly MJ, Green T et al: Absence of linkage between inflammatory bowel disease and selected loci on chromosomes 3, 7, 12, and 16. Gastroenterology 1998; 115: 1062-1065.

16 Curran ME, Lau KF, Hampe J et al: Genetic analysis of inflammatory bowel disease in a large European cohort supports linkage to chromosomes 12 and 16. Gastroenterology 1998; 115: 1066-1071.

17 Lennard-Jones JE: Classification of inflammatory bowel disease. Scand J Gastroenterology 1989; 24 (suppl. 170): $2-6$.

18 Sambrook J, Fritsch EF, Maniatis F: Molecular Cloning: A Laboratory Manual. Cold Spring Harbor Laboratory Press: Cold Spring Harbor, New York, 1989.

19 Dib C: A comprehensive genetic map of the human genome based on 5,264 microsatellites. Nature 1996; 380: $152-154$.

20 Annese V, Piepoli A, Andriulli A et al: Polymorphism of motilin gene in patients with Crohn's disease. Dig Dis Sci 1998; 43: 715-719.

21 Kruglyak L, Daly MJ, Reeve-Daly MP, Lander ES: Parametric and nonparametric linkage analysis: a unified approach. Am J Hum Genet 1996; 58: 1347-1363.

22 Terwilliger JD: A powerful likelihood method for the analysis of linkage disequilibrium between trait loci and one or more polymorphic market loci. Am J Hum Genet 1995; 56: 777-787.

23 Lander ES, Kruglyak L: Genetic dissection of complex traits: guidelines for interpreting and reporting linkage results. Nat Genet 1995; 11: 241-247.

24 Krugklyak L, Lander ES: High resolution genetic mapping of complex traits. Hum Genet 1995; 57: 1212-1223.

25 Nair RP, Henseler T, Jenish S et al: Evidence for two psoriasis susceptibility loci (HLA and 17q) and two novel candidate regions (16q and 20p) by genome-wide scan. Hum Mol Genet 1997; 6: 134913-134956.

26 Lee FI, Bellary SV, Franci C: Increased occurrence of psoriasis in patients with Crohn's disease and their relatives. Am J Gastroenterol 1990; 85: 922-923. 
27 Satsangi J, Welsh HI, Bunce M et al: Contribution of genes of the major histocompatibility complex to susceptibility and disease phenotype in inflammatory bowel disease. Lancet 1996; 347: 1212-1217.

28 Roussomoustakaki M, Satsangi J, Welsh KM et al: Genetic markers may predict disease behavior in patients with ulcerative colitis. Gastroenterology 1997; 112: 1845-1853.
29 Perri F, Annese V, Piepoli A et al: HLA antigens and pANCA define ulcerative colitis as a genetically heterogeneous disorder. It J Gastroent Hepatol 1998; 30: 56-61. 